III. SMALL-SCALE MAGNETIC FIELDS 


\title{
EMPIRICAL MODELS OF PHOTOSPHERIC FLUX TUBES
}

\author{
S.K. SOLANKI \\ Department of Mathematical Sciences, \\ Unversity of St Andrews, \\ St Andrews, KY16 9SS, \\ Scotland
}

\begin{abstract}
The empirically derived properties of magnetic flux tubes at both ends of the size spectrum, i.e. magnetic elements and sunspots, are reviewed. Emphasis is placed on quantitative results. The following parameters are discussed in greater detail: The strength and structure of the magnetic field, the temperature stratification and the structure of the velocity field.
\end{abstract}

\section{Introduction}

The concept of flux tubes has been successfully used to describe solar photospheric magnetic structures as diverse as sunspots (penumbral diameters generally larger than $5000 \mathrm{~km}$ ) and magnetic elements (diameters less than approximately $300 \mathrm{~km}$ ). A photospheric flux tube can be roughly described as a bundle of field lines passing with a more or less vertical axis through the non-magnetic solar photosphere, although highly inclined flux tubes can also be envisaged, for example during flux emergence and submergence.

This review covers some of the quantitative observational results obtained on these structures. Although I have tried to treat both sunspots and magnetic elements in equal detail, an imbalance is unavoidable, due to my restricted experience with sunspots. A lack of space forces the exclusion of flux tubes of intermediate size, like pores or magnetic knots. Also, at present it is still unclear whether the latter are really single flux tubes, or whether they are conglomerates of smaller flux tubes (cf. Knölker and Schússler, 1988). The present review also does not cover possible "weak" mixed polarity fields, since their nature has not yet been resolved and it is not known whether the flux tube picture applies to them. For more details on such features see Stenflo $(1988,1989)$ and Martin (1989 and these proceedings).

Even within the domain of small scale concentrated fields, there are a number of important topics not covered by this review. For example, it does not deal with those aspects of small scale magnetic fields reviewed elsewhere in these proceedings. Thus I shall leave theoretical aspects and a description of our basic physical understanding of these structures almost entirely to Schüssler and Ryutova, the discussion of their evolution, as derived from magnetograms, to Martin, and questions of morphology to Muller and Title. Before starting on the three main topics, strength and structure of the magnetic field, thermal stratification and velocity structure, let me make a few remarks on the modelling of solar magnetic features in general.

\section{General Remarks on the Empirical Modelling of Magnetic Features}

There are three main approaches to determining the atmospheric structure of solar magnetic features.

a) Purely empirical: As many quantities as possible are individually and independently derived from the observations. For those quantities for which no direct observational information is available, "reasonable" values are assumed.

b) Semi-empirical: Key quantities are determined from observations, but the remaining parameters are 
derived self-consistently from these using simple physics (e.g., hydrostatic equilibrium and thin tube approximation). Semi-empirical models are generally derived iteratively through a succession of steps. Synthetic spectra are compared with observed spectra and the model is changed until the discrepancies become sufficiently small.

c) Ab initio: All magnetic and hydrodynamic quantities are derived from basic physical laws, with a minimum of initial observational input. Clues to missing physics or inapropriate assumptions can be obtained by comparing with semi-empirical models, or directly with observations.

Each of the three approaches has a place within the study of solar magnetic features. However, the emphasis has been shifting from purely empirical to the semi empirical and the purely theoretical approaches, as the data and our idea of the underlying physics continue to improve.

All empirical models are no better than the data they are based on and, even more restrictively, the exact diagnostic used. It is, therefore, crucial to develop good diagnostics which are, ideally, very sensitive to a given model parameter and insensitive to all other model parameters. Unfortunately, ideal or nearly ideal diagnostics are rare, so that generally many different diagnostics must be applied simultaneously to complement each other and constrain the structure of the magnetic feature sufficiently. In practice, this often implies the use of more spectral lines. However, only in very few cases can a model be completely prescribed by the observations and additional physical input is generally required. For this reason the semi-empirical approach is generally to be preferred to the purely empirical approach.

There are broad parallels between the construction of empirical models for magnetic elements and sunspots, particularly the need for multi-component, or 2-D models.

Unpolarized low spatial resolution data from network elements and active region plages or faculae can be reproduced by single component models. However, high resolution and polarimetric data show that the field is concentrated into small elements surrounded by a relatively field free atmosphere, calling for a 2-component model: A magnetic component covering a fraction $\alpha$ of the surface within the spatial resolution element of the telescope, and a non-magnetic component covering a fraction $1-\alpha$, where $\alpha$ is the magnetic filling factor. In such a model the observed Stokes parameters, averaged over both components $(\langle I\rangle=$ unpolarized light, $\langle Q\rangle$, $\langle U\rangle=$ difference between two orothoganal linear polarizations, $\langle V\rangle=$ difference between the two senses of circular polarization) can be written as:

$$
\langle I\rangle=\alpha I_{\mathrm{m}}+(1-\alpha) I_{\mathrm{nm}}, \quad\langle Q\rangle=\alpha Q_{\mathrm{m}}, \quad\langle U\rangle=\alpha U_{\mathrm{m}}, \quad\langle V\rangle=\alpha V_{\mathrm{m}} .
$$

$I_{\mathrm{m}}, Q_{\mathrm{m}}, U_{\mathrm{m}}$ and $V_{\mathrm{m}}$ are the Stokes profiles emanating from the magnetic feature, while $I_{\mathrm{nm}}$ is the only Stokes parameter with a contribution from the non-magnetic region in the resolution element. Stokes $V, Q$ and $U$ are totally independent of the non-magnetic component. Relatively unequivocal information on the magnetic features can be obtained if at least one of these three parameters is observed. Due to the often large linear polarization produced by most solar telescopes, Stokes $V$ has traditionally been used the most, although all four Stokes profiles are required to determine the full magnetic field vector. As we shall see, even at disk centre some observations require proper 2-D models of magnetic elements due to their rapid expansion with height. Closer to the limb 2-D becomes an absolute must (Van Ballegooijen, 1985a, b), since one of the basic assumptions of empirical two- or multi-component models breaks down. Due to the small size of magnetic elements and their expanding canopy a single ray may pass through more than one component, so that the various components are no longer diagnostically decoupled. By this I mean that spectra cannot be calculated individually in each component and the averaged spectrum is no longer simply a sum of the suitably weighted spectra from the individual components.

Although single component models of sunspot umbrae have been quite successful in reproducing low spatial resolution continuum data, the presence of spatially unresolved small-scale structure (umbral dots) also requires the construction of 2-component models of the umbra if higher resolution observations are to be reproduced, with one component each for the dark and the bright features. Due to the small size of umbral dots, models based on data obtained away from disk centre should be two-dimensionsal, since some rays pass through both atmospheric components and the assumption of decoupled atmospheres breaks down. The highly structured penumbra, obviously, also requires at least multi-component models.

Note that since all components of sunspots are permeated by a magnetic field, all four observed Stokes parameters now obtain contributions from both the bright and dark features. If we assume these to cover a 
fraction $\alpha$ and $(1-\alpha)$ of the resolution element, respectively, then the observed Stokes parameters can be written as

$$
\langle I\rangle=\alpha I_{\mathrm{b}}+(1-\alpha) I_{\mathrm{d}}, \quad\langle Q\rangle=\alpha Q_{\mathrm{b}}+(1-\alpha) Q_{\mathrm{d}}, \quad\langle U\rangle=\alpha U_{\mathrm{b}}+(1-\alpha) U_{\mathrm{d}}, \quad\langle V\rangle=\alpha V_{\mathrm{b}}+(1-\alpha) V_{\mathrm{d}}
$$

Subscripts ' $b$ ' and ' $d$ ' denote the bright and dark components respectively. Some of the main advantages of observing Stokes $Q, U$, or $V$ are therefore lost when studying sunspot fine structures (although the problem of stray light is substantially reduced). This makes the empirical determination of sunspot fine structure properties an extremely difficult task.

\section{Magnetic Field}

\subsection{Sunspots}

The resolvable, large scale structure of the sunspot magnetic field strength is relatively straightforward to measure (e.g. from the wavelength difference between the two $\sigma$-components of a completely Zeeman split line, although more refined techniques have also been applied) and has been determined by a number of authors with relatively high accuracy. A comparison between the results of different authors can best be made for large and relatively symmetrical sunspots. Mattig (1961), Stepanov (1965), Ioshpa and Obridko (1965), Beckers and Schröter (1969), Wittmann (1974), Gurman and House (1981), Kawakami (1983) and Lites and Skumanich (1989) have, amongst others, derived the field strength as a function of distance from the centre of such sunspots. A selection of $B(r)$ dependences are plotted in Fig. 1.

The field strength near the sunspot centre can vary from sunspot to sunspot, but generally lies in the range 2000-3000 G. In particular, Brants and Zwaan (1982) have shown using purely umbral lines (i.e. lines which are much stronger in the umbra than in the photosphere) that no sunspot umbra, not even the smallest, has a central field strength lower than $2000 \mathrm{G}$ which is considerably higher than values derived from lines also present in the penumbra and the quiet photosphere. Due to the presence of fine scale structure in both the umbral (light bridges, umbral dots) and the penumbral photospheres (penumbral filaments, penumbral grains), the field strengths derived above are averages over the field strengths in the bright and dark components.

The final aim is to obtain the complete magnetic field vector at all points in space above a sunspot. Important contributions have been made by, among others, Beckers and Schröter (1969) (only from Stokes $I$ and V), Deubner and Göhring (1970), Wittmann (1974), Gurman and House (1981), Hagyard et al. (1983), Kawakami (1983) and Lites and Skumanich (1989). Ioshpa and Obridko (1965), Hagyard et al. (1983), and Lites and Skumanich (1989) find that the observed field is close to potential, although Wittmann (1974) and Lites and Skumanich (1989) do find evidence for a twist around the vertical axis in some symmetric sunspots, even after taking magnetooptical effects into account. Fig. 2 shows the inclination angle of the field with respect to the vertical as a function of the normalised sunspot radius, as derived by various authors. One interesting result of the most recent determinations is that the field in the penumbra is not completely horizontal, not even at the outermost edge. However, seeing fluctuations and the unresolved penumbral fine structure may affect this result.

The determination of the field strength variation over the fine scale structure in the sunspot umbra and penumbra is a difficult undertaking and widely different results have been published. Empirical estimates of field strengths in umbral dots range from: $10 \%$ of the field strength in the dark umbral core (with opposite polarity) by Beckers and Schröter (1969) (A misinterpretation of magnetooptical effects? See Wittmann, 1971), $50 \%$ by Obridko (1968) and Kneer (1973), $90 \%$ by Buurman (1973), Adjabshirzadeh and Koutchmy (1983) and Pahlke (1988) and, finally, Zwaan et al. (1985) and Lites and Scharmer (1989), who find no evidence of a weaker field in umbral dots. Although the newer observations favour higher $B$ values, the problem is not yet completely resolved, mainly due to its intractability (see end of Sect. 2).

In the penumbra Beckers and Schröter (1968) and Abdussamatov (1976) found 100-400 G higher $B$ values in dark penumbral filaments than in bright ones from direct high spatial resolution observations. We are also fortunate that the $12 \mu$ lines are observed in emission in the penumbra. The discovery of the great Zeeman sensitivity of these lines (they are approximately 7-8 times more sensitive than Fe I $5250.2 \AA$ ) by 


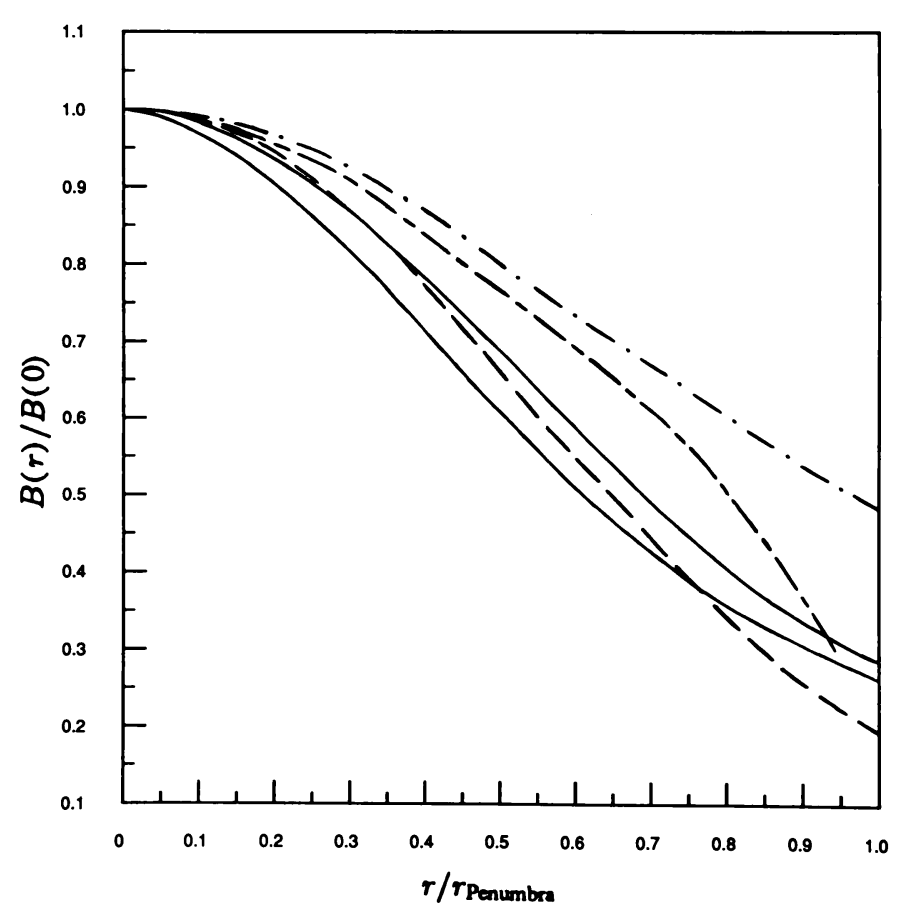

Fig. 1: Magnetic field strength normalised to the central field strength $B(r) / B(0)$, of large symmetrical sunspots vs. radial distance from sunspot centre normalised to the outer penumbral radius, $r / r_{\text {Penumbra }}$. Dot-dashed curve: Beckers and Schröter (1969), long and short dashes: Wittmann (1974); dashed curve: Kawakami (1983); Solid curves: two sunspots studied by Lites and Skumanich (1989). For the spots observed by Lites and Skumanich the umbral radius corresponds to $r / r_{\text {Penumbra }}=0.4$.

Brault and Noyes (1983) and their identification by Chang and Noyes (1983) has provided us with an exciting new diagnostic of $B$ near the height of the photospheric temperature minimum. Deming et al. (1988) have compared the broadening of the $\sigma$-components of a $12 \mu \mathrm{Mg}$ I line with the width of its $\pi$-component. Since the $\sigma$-components are considerably broader and the vertical $B$-gradient is expected to be relatively small, they conclude that there is a distribution of field strengths with a full width at half maximum of approximately $450 \mathrm{G}$. Note, however, that due to uncertainties in the formation mechanism of the line, this value is not a stringent limit. If this line is temperature sensitive, then it may be formed preferentially in one of the penumbral components, so that the field strength may differ by more than $450 \mathrm{G}$. On the other hand, if part of the broadening is due to inhomogenieties in the Evershed flow (cf. Sect. 5.1), then the field strength variation may be smaller than $450 \mathrm{G}$.

At least a part and perhaps all of the horizontal variation of the field strength between bright and dark features in umbrae and penumbrae may be caused by different heights of formation of the spectral lines in these features. It is premature to talk of inhomogenieties in the actual magnetic field before this point has been settled.

\subsection{Magnetic Elements}

Since magnetic elements have generally not been spatially resolved and their field strength is insufficient to completely split spectral lines in the visible, indirect techniques have had to be developed to measure the true 


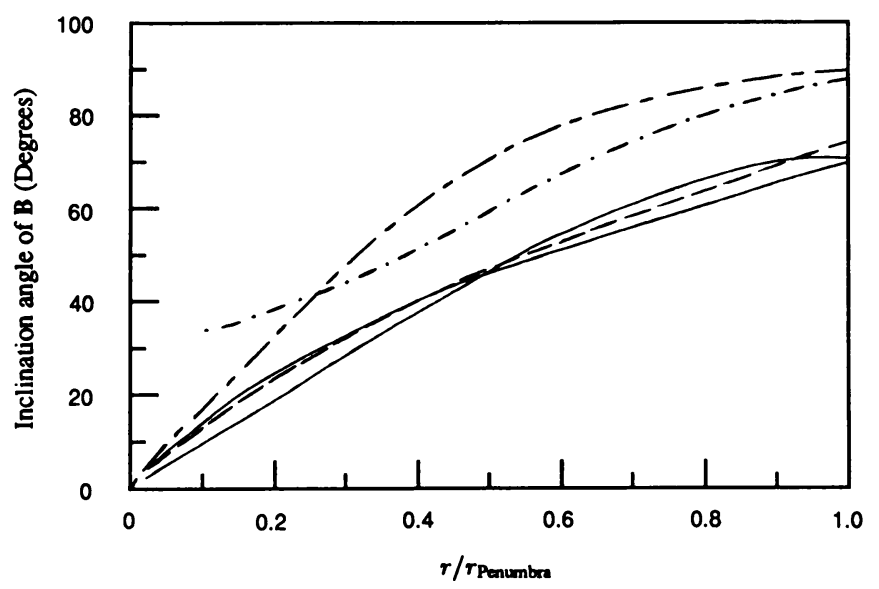

Fig. 2: Inclination angle to the vertical of the magnetic field vector vs. $r / r_{\text {Penumbra }}$. The various curves correspond to the same measurements as in Fig. 1.

field strength in such structures. "Field strengths" derived without such techniques reflect not only the intrinsic field strength, but also depend on the magnetic filling factor (which is influenced by the spatial resolution achieved by the observations), on the assumptions made for the atmospheric structure of the magnetic elements and, for Stokes $I$ observations, their surroundings. I begin by reviewing the field strength measured at a single height in the photosphere.

The first relatively model independent diagnostic, the so-called line-ratio technique, was introduced by Stenflo (1973). It is based on the comparison of the Stokes $V$ profile of a line having a large Lande factor to that of a line with a smaller Landé factor. Relative model independence is achieved if both lines have similar line strengths, excitation potentials and wavelengths. For sufficiently weak fields the ratio of the amplitudes of the two Stokes $V$ profiles is simply proportional to the ratio of their Lande factors, while for sufficiently strong fields the ratio is unity (when both lines are completely split). However, for fields of intermediate strength $(0.5-2.0 \mathrm{kG})$ the ratio of the two Stokes $V$ profiles varies as a function of field strength (Zeeman saturation effect). The measurement of this line-ratio is, therefore, a straightforward diagnostic of field strength, although the exact calibration generally requires some model calculations.

This technique has been extended and applied by Frazier and Stenflo (1978), Wiehr (1978), Stenflo and Harvey (1985), Rachkovsky and Tsap (1985), Solanki et al. (1987), Sánchez Almeida et al. (1988b) and Lozitskij and Tsap (these proceedings). Although the results of Wiehr (1978) and Lozitskij and Tsap differ slightly from the others, the basic result is that at the heights at which the Stokes $V$ profiles of lines in the visible are formed (i.e. the middle photosphere) the field strength averaged over the flux tube cross-section is approximately $1000-1200 \mathrm{G}$.

In the mean time a host of other techniques has also been developed, most of which, unfortunately, are of lower accuracy. Indirect support for $\mathrm{kG}$ fields has come from empirical flux tube models. Thus, Chapman (1974) found that a field strength of approximately $1800 \mathrm{G}$ is required to obtain consistency within his empirical flux tube model. Similarly Koutchmy and Stellmacher (1978) derived field strengths of approximately $1500 \mathrm{G}$ by comparing observations of $I+V$ and $I-V$ with model calculations. Tarbell and Title (1977) applied a technique based on the Fourier transform of the Stokes $V$ profile (under some assumptions on the profile shape) to solar data and derived field strengths between $1000 \mathrm{G}$ and $1800 \mathrm{G}$. Robinson et al. (1980) and later Sun et al. (1986) applied a technique developed by Robinson (1980) which is based on the Fourier transform of the Stokes $I$ profile and which has had its main application to the detection of fields on late type stars (cf. Saar, these proceedings). They also generally found $\mathrm{kG}$ fields with a couple of exceptions. However 
Saar (1988) and Hartmann (1987) have shown that in the simple form used by the above authors this techniqe can give wrong values of $B$. Solanki and Stenflo (1984) derived a kG intrinsic field strength from a statistical analysis of a large number of Stokes $V$ profiles of mostly weak Fe I lines. Recently Del Toro Iniesta et al. (1989) have extended the centre of gravity technique (e.g. Rees and Semel, 1979). When applying it to data of approximately $1^{\prime \prime}$ resolution they find field strengths between $550 \mathrm{G}$ and $1700 \mathrm{G}$, with a preponderance of lower field strengths. They suggest that the difference between their results and those obtained from other sources is due to differences in spatial resolution. On the other hand, Keller et al. (1989) have applied the line ratio technique to data with a spatial resolution of $0.5^{\prime \prime}-1^{\prime \prime}$ and find that the observations are compatible to a kG field strength in all the features. They show that the scatter in the observed line ratio is explained in a natural manner by noise in the data. It may be concluded from the above that, given the fact that not all techniques are equally reliable, the results are relatively consistent: The field strength in the middle photosphere is between 1000 and $1500 \mathrm{G}$.

The main advances regarding our knowledge of the magnetic field structure have, in recent years, come from observations in the infrared. The $12 \mu$ lines have been exploited by Brault and Noyes (1983), Deming et al. (1988) and Zirin and Popp (1989). In active regions (but outside sunspots) $B \approx 250-550 \mathrm{G}$ is found near the temperature minimum, although the exact height of formation of these lines within magnetic elements is all but clear and even their formation mechanism is not beyond doubt (cf. Lemke and Holweger, 1987). However, they are probably formed in either the upper photosphere or lower chromosphere and the smaller field strengths observed in them do imply a general decrease of field strength with height, as expected from pressure balance.

At present it is simpler to map the derived magnetic field to a certain height by using lines formed at shorter wavelengths in the infrared, for example, the Fe I $g=3$ line at $1.56485 \mu$. Harvey and Hall (1975) first noted that this line is three times more Zeeman sensitive than, e.g., the $g=3 \mathrm{Fe}$ I $5250.2 \AA$ line and derived kG field strengths. Stenflo et al. (1987b), Solanki et al. (1989) and Zayer et al. (1989) confirmed and refined the findings of Harvey and Hall (1975), showing that at the height of formation of this line (lower photosphere) the field strength is approximately $1500-1600 \mathrm{G}$. This very sensitive and model independent diagnostic, therefore, fully confirms the predominance of $\mathrm{kG}$ fields in the lower and middle photosphere. From the profile of this line, and by comparing it with a low $g$ line in the infrared as well as with lines in the visible formed higher in the atmosphere, Solanki et al. (1989) and Zayer et al. (1989) obtained a good idea of the vertical and horizontal structure of the magnetic field strength in the lower and middle photosphere. A 2-D model containing a field with $B=2000 \mathrm{G}$ at $\tau=1$ which is stratified according to exact pressure balance, so that it drops approximately exponentially with height, reproduces the observational constraints on the field strength in the lower and middle photosphere to a high degree. Their analysis also implies that in the lower photosphere, in contrast to sunspots, the field strength is horizontally constant within the tube with a relatively sharp boundary to the non-magnetic atmosphere. The observations, therefore, favour flux tube models of magnetic elements having a boundary current sheet.

The determination of the full magnetic vector is still at an early stage and the reliability of the measurements carried out so far and their interpretation may, perhaps, be questioned. Due to their limited spatial resolution, observations can so far only give an idea of the inclination of whole groups of magnetic elements. Deubner (1975) mentions that he finds evidence of an almost random distribution of field line inclinations. Solanki et al. (1987) derived limits to the inclination from Stokes $I, Q$ and $V$ line profiles and found that the magnetic elements averaged over several thousand $\mathrm{km}^{2}$ on the solar surface can be inclined in a preferred direction by more than $10^{\circ}$ to the vertical. Lites and Skumanich (1989) support these findings.

\section{Thermal Structure}

\subsection{Sunspots}

The empirical modelling of the thermodynamical structure of sunspot umbral photospheres is a mature subject, with a large body of work. I shall, therefore, concentrate mainly on more recent investigations.

One problem facing sunspot modellers is that properties of sunspots may depend on their size, shape, age and even on the phase of the solar cycle. If so, then no unique model may be able to describe all sunspots 
or even all stages in the life of a single sunspot. Maltby et al. (1986) argue that there is no evidence of variations in dark sunspot umbral cores from one sunspot to another, except with the solar cycle (Albregtsen and Maltby, 1978). Also, Sobotka (1988) finds no dependence of the temperature on age of small sunspots, but, on the other hand, he does see the need of different umbral dot filling factors in different umbrae. Finally, Grossmann-Doerth et al. (1986) find differences between the properties of umbral dots in different parts of the umbra.

As a way out of this dilemma modellers have either restricted the applicability of their models to, e.g., large sunspots (Maltby et al., 1986; Obridko and Staude, 1988) or have presented different models, e.g., for different sunspot sizes and different umbral dot fillings (Sobotka, 1988), or for different phases of the solar cycle (Maltby et al., 1986).

If inhomogenieties like umbral dots are ignored, or only that part of the umbra which appears to be uniform is considered, then single component models can be constructed. Such models exist in profusion and now rival the quiet photosphere models with regard to the breadth of data which they are based on. Recent models of the darkest part of the umbral photosphere have been constructed by Kollatschny et al. (1980) and Van Ballegooijen (1984) which are based on line profiles, and by Maltby et al. (1986), based on purely continuum data. These models are relatively similar and satisfy the observations almost equally well. Although the latter authors do not see any signs for the need of two-components, Van Ballegooijen finds such a need, possibly because he is fitting line profiles which can be more sensitive to inhomogenieties.

A number of authors have taken the alternative approach and have constructed 2-component models of umbrae, with a cold component describing the umbral core, and a warmer component describing umbral dots. Perhaps a third component describing light bridges in old or complex sunspots should also be introduced. Specific models for light bridges have been made by Sobotka (1989).

Adjabshirzadeh and Koutchmy (1983) and Obridko and Staude (1988) have published 2-component models. The most comprehensive is that of the latter authors which satisfies a wide variety of observational data on large sunspots. Among other things its cold component reproduces the continuum observations of Albregtsen et al. (1984), on which the deeper layers of the Maltby et al. model are based, while a $50 \%$ mixture of the hot component and the cold component can reproduce the umbral dot brightness observations of Wiehr and Stellmacher (1985). For smaller sunspots Sobotka (1988) has proposed 2-component models based on a few spectral lines. These are simpler than the models described above, being "down-scaled" photospheric models. He finds large variations in the filling factors of the bright component of small umbrae. Whether this is due to the rapidly increasing problem of stray light with decreasing umbral diameter is unclear. The temperature stratification of a few recent umbral models is illustrated in Fig. 3.

The temperature of umbral dots is still a debated subject, with two schools of thought. The first claims that dots have photospheric brightness and hence temperature (e.g. Beckers and Schröter, 1968; Koutchmy and Adjabshirzadeh, 1981; Pahlke, 1988), while the other suggests that they are a few 100 to $1000 \mathrm{~K}$ cooler than the quiet photosphere, depending partly on their position in the umbra (e.g. Grossmann-Doerth et al., 1986; Obridko and Staude, 1988; Sobotka, 1988). Since umbral dots are generally spatially unresolved and both umbral components have a magnetic field, the derivation of true dot temperatures is an unresolved issue. Indirect techniques, such as using Stokes $V$ profiles of lines with different excitation potentials (Pahlke, 1988) can only reduce the problem, but not resolve it. In many ways the problem is similar to that of deriving the temperature structure of magnetic elements from Stokes $I$ profiles (see Sect. 4.2). The disadvantages mentioned in that connection largely also apply here.

The penumbra is possibly the most visible example of a solar photospheric structure requiring multicomponent models. In white light the pattern of bands of varying shades of brightness is quite striking. Krat et al. (1972) and Muller (1973) applied the 2-component approach to the brightness distribution of the penumbra and sorted the penumbral filaments into bright and dark bins with approximately $0.7-1$ and $0.3-0.7$ times the photospheric brightness, respectively. Kjeldseth-Moe and Maltby (1974) used these continuum data to derive 2-component models of the penumbra which, when appropriately combined, also reproduce low resolution multi-wavelength continuum observations obtained by Maltby (1972). Their model has been included in Fig. 3. Although a simple 2-component scheme has been called into question by Grossmann-Doerth and Schmidt (1981), who point out that the histogram of the continuum intensities of penumbral filaments is single peaked (with brightness ranging between approximately 0.5 and 1.0 times the photospheric value), a 2-component 


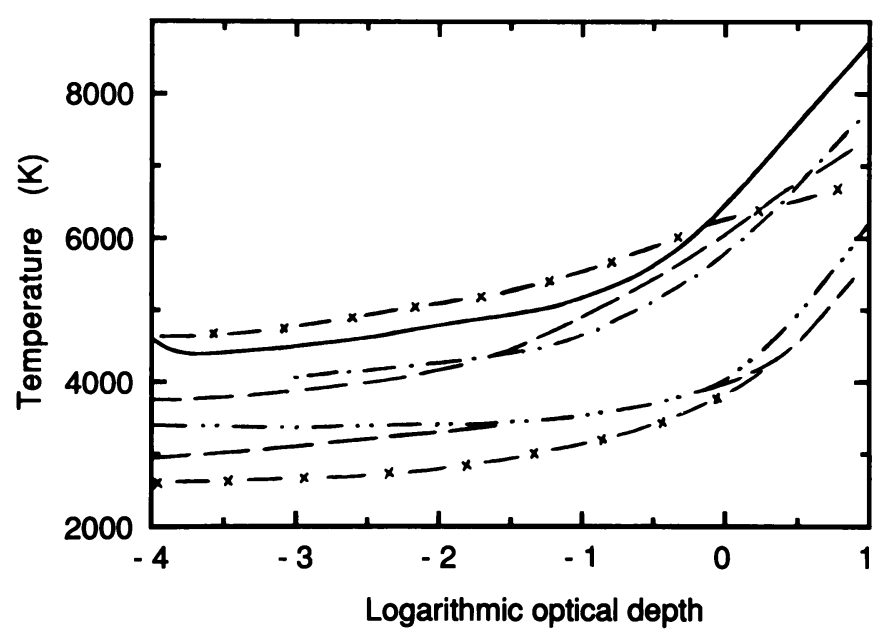

Fig. 3: Temperature vs. logarithmic optical depth of a selection of models of various sunspot components and of the quiet sun (solid line). Maltby et al. (1986) umbral core model: __ . _ (1988) umbral core and umbral dot models: ___ - Adjabshirzadeh and Koutchmy (1983) umbral core and dot models: — $\_-$; Kjeldseth-Moe and Maltby (1974) dark penumbral filament model: —- (their bright penumbral filament model is similar to the quiet photosphere model and has not been plotted separately).

approach is a first step on the way towards reliable empirical penumbral models. However, the caveat of Grossmann-doerth and Schmidt (1981) demonstrates tha compared to umbrae, penumbral modelling is still at an early stage and high quality, high resolution data are very desirable.

\subsection{Magnetic Elements}

Empirical models of magnetic features outside of sunspots also have a long and varied history. A plethora of 1-component, later 2-component and recently 2-D models have been constructed with the help of data ranging from the centre to limb variation (CLV) of continuum contrast measurements at one wavelength to Stokes $V$ profiles of many lines.

The oldest types of models are one-component models based on low spatial resolution data. Such models describe some ill-defined average over the magnetic elements and their surroundings. They generally have a temperature very similar to the quiet sun at equal optical depth near $\tau=1$ at $5000 \AA$ and a gently increasing temperature difference to the quiet sun with height throughout the photosphere (i.e. decreasing optical depth, cf. Model by Shine and Linsky, 1974, in Fig. 4). Such models have been constructed by, e.g., Schmahl (1967), Stellmacher and Wiehr (1973), Shine and Linsky (1974) and Vernazza et al. (1981, model F). The general $T(\tau)$ shape of such models may easily be guessed by simply looking at an image of the solar disk in white light: Faculae are not seen near the centre of the disk, but are clearly visible as bright features near the limb, where the continuum is formed higher in the atmosphere. In summary, as long as the spatial resolution is low, single component models are relatively well defined, although they do not describe any part of the real solar atmosphere in active regions.

In contrast to this, the results of 2-component modelling depend very strongly on the data and, in some cases, on additional assumptions. We can divide such models into basically two groups, those based on Stokes $I$ and those based on Stokes $V$ (and, in future, possibly Stokes $Q$ and $U$ as well, see Solanki et al., 
1987). Both groups share one major uncertainty, namely the value of the continuum intensity near disk centre, which determines the lower photospheric layers of the models. However, whereas models can be derived from Stokes $V$ with a minimum of additional assumptions (if the correct combination of lines is used), the approach based on Stokes $I$ requires another two major assumptions: a) the filling factor of the magnetic features in the resolution element, b) the temperature of the atmosphere surrounding the magnetic elements. Recall that Stokes $I$ obtains contributions from both magnetic and non-magnetic components.

Before discussing the various types of models, let me briefly discuss the continuum contrast observations, i.e., the ratio of the continuum intensity in the magnetic elements to that in the quiet sun. The directly observed continuum contrast is one of the most uncertain parameters of magnetic elements. Values range from 1 to 2 and depend on the spatial resolution of the observations. Higher resolution observations give larger contrasts (compare Koutchmy, 1977; Muller and Keil, 1983; Foukal and Fowler, 1984; Hirayama et al., 1985, etc.). Schüssler and Solanki (1988) introduced another technique, based on the line ratio of two Stokes $I$ profiles, which specifically takes the unknown temperature of the surroundings into account. After assuming an upper limit of $25 \%$ for the filling factor of their observed region they were able to set a lower limit of 1.4 on the continuum contrast. Particularly exciting are speckle observations which allow very high spatial resolution to be achieved and which show points, associated with $\mathrm{Ca}$ II brightenings and therefore probably with magnetic fields, that are around 1.5 times brighter than the quiet sun (Von der Lühe, 1989). The difference between low and high spatial resolution results can best be reconciled in active regions if the continuum forming layers of the non-magnetic atmosphere between magnetic elements is cooler than the quiet sun by approximately 100 K (Schüssler and Solanki, 1988). This lower temperature has been confirmed using other data and another technique by Brandt and Solanki (1989).

One single component and a selection of 2-component models derived from Stokes $I$ measurements are plotted in Fig. 4. They show tremendous differences from one to another. I shall now try to roughly explain the main features of these models.

All the plotted 2-component models have an appreciably higher temperature in the middle and upper photosphere than the quiet sun and the 1-component model. The reason is that now the observed line weakening (or, in some models, the continuum contrast enhancement near the limb) is assumed to be produced entirely within the small fraction of the surface covered by the magnetic elements. Note that the modellers using Stokes $I$ data have assumed the magnetic elements to be surrounded by the quiet sun. Around the height where the lines are formed $(-2.5 \lesssim \log \tau \lesssim-1.5)$ the 2-component models are reasonably similar, given the uncertainty in filling factor. The difference between the dashed (Chapman) and the double dashed (Walton) curves in Fig. 4 illustrates the effect on the derived stratification of choosing different filling factors, since Walton (1987) also obtained a model very similar to Chapman's simply by increasing the assumed value of $\alpha$. Some of the differences between models in the upper layers may be solar (e.g. Hirayama, 1978, derived two different models from his continuum contrast data, of which only the hotter is shown, see also the discussion of the Stokes $V$ based models, below). However, the models differ substantially in the deeper layers where they rely on continuum contrast near solar disk centre. Models based on low spatial resolution, i.e., low continuum contrast data (e.g. Chapman, 1970, 1977, 1979; Walton, 1987) usually have a temperature close to the quiet sun value in the deeper layers, while models based on high spatial resolution, i.e., high continuum contrast data (e.g. Koutchmy and Stellmacher, 1978; Stellmacher and Wiehr, 1979), have a high temperature in their lower layers.

The Stokes $I$ models therefore reflect mainly 1) the data they are based on, 2) the choice of the modeller regarding filling factor and external atmosphere and 3) partly real differences between solar magnetic features. The general features of the Stokes $I$ models can, therefore, be understood if only a few particulars are kept in mind.

There are fewer models based on Stokes $V$. The first Stokes $V$ based model was that of Stenflo (1975) derived from a few lines and assuming a continuum contrast close to unity which was an accepted value at that time. Consequently it differs considerably in its lower layers from the more recent models of Solanki (1986) and Keller (these proceedings). However, at the level of line formation it is quite similar (cf. Fig. 5). The models of Solanki satisfy selected line parameters of Stokes $V$ profiles of hundreds of simultaneously observed unblended Fe I and II lines. However, they are not based on a least squares fit (inversion technique). The models derived by Keller (these proceedings) have been calculated both in 1-D and 2-D (which produce 


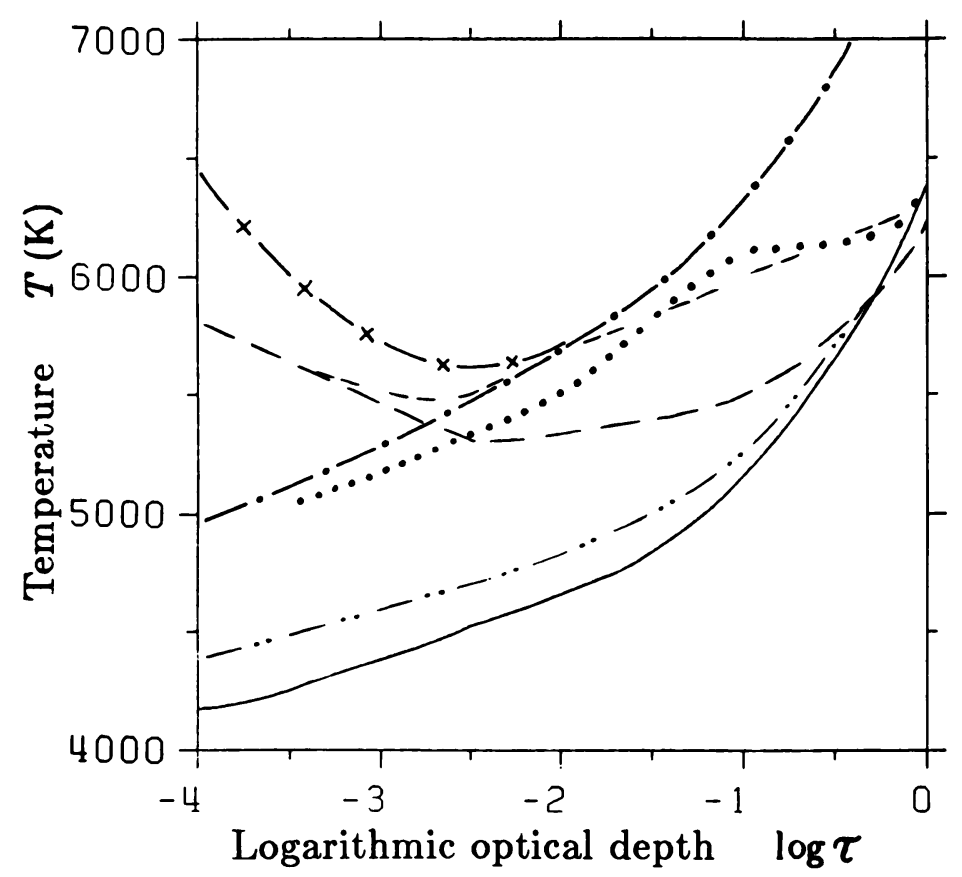

Fig. 4: Temperature of facular models based on Stokes $I$ vs. logarithmic optical depth. Quiet sun (HSRA, Gingerich et al., 1971): solid line. 1-component model of Shine and Linsky: _ . - ; 2-component models of Chapman (1977): ——— Walton (1987): - — - -; Hirayama (1978): . . . . ; Koutchmy and Stellmacher (1978): $\_\times —$; Stellmacher and Wiehr (1979): $\_-. \longrightarrow$.

almost identical temperature stratifications). They are based on a smaller data set (10 lines), but, on the other hand, they have been derived using an "inversion" of these data, i.e. these models give the best least squares fit to the 10 Stokes $V$ profiles from the starting values chosen.

Despite the large data sets underlying them, these models are only reliably determined from Stokes $V$ between approximately, $-3 \lesssim \log \tau \lesssim-1$. In particular, below $\log \tau \approx-1$ the models are mainly determined by the continuum intensity. This has been taken to lie around 1.3-1.4 by Solanki (1986) and at 1.8 by Keller. Both Solanki (1986) and Keller (these proceedings) derive different models for active region plages and the network with flux tubes being warmer when the filling factor is lower. Although this result was originally based on observations of only a few regions, the work of Pantellini et al. (1988) and more recently Zayer et al. (to be published) has confirmed this trend for many regions covering a large range of filling factors.

The models of Keller (these proceedings) are reasonably similar to the models of Solanki (1986) in the height range over which they are reliable, which is not all too surprising since they are based on partially the same data. The models by the above-mentioned authors based on data from the enhanced network differ from each other more than those based on the plage data due to the lower $\mathrm{S} / \mathrm{N}$ in the network data.

In summary, the models based on Stokes $V$ overcome some of the main disadvantages faced by purely Stokes $I$ based models, and also appear to be reasonably reliable, within their limitation. The three main limitations are: 1) The current models are based on relatively low resolution data, so that a single model may be trying to describe magnetic elements having different properties. 2) All current models rely on continuum contrast measurements for the determination of the temperature in the deeper photospheric layers. 3) LTE 


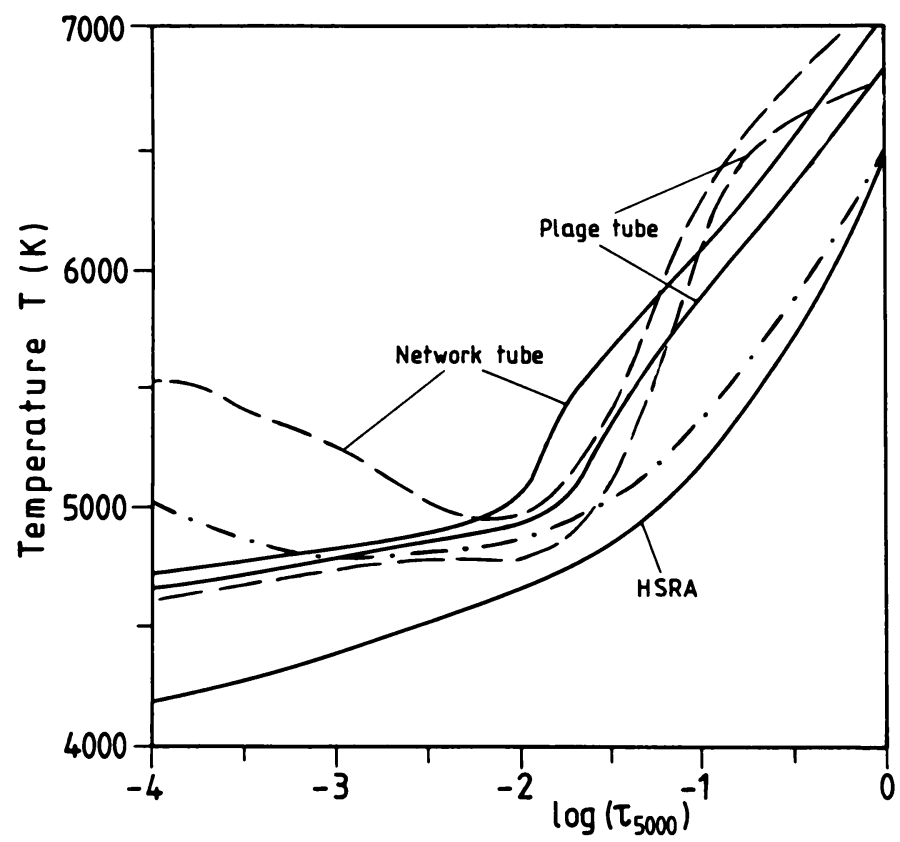

Fig. 5: Temperature of magnetic element models based on Stokes $V$ vs. logarithmic optical depth, compared to the model of the quiet sun, the HSRA (marked). Stenflo (1975): dot-dashed curve; Solanki (1986): solid curves; Keller (these proceedings): dashed curves. Where separate models for the network and for plages exist these have been marked.

is assumed. The importance of NLTE effects in a 2-D flux tube with hot walls has been demonstrated by Stenholm and Stenflo (1978) using a 2-level atom. Solanki and Steenbock (1988), on the other hand, used only a 1-D flux tube model, but a very comprehensive iron atomic model and an empirical temperature stratification to show that departures from LTE are larger in magnetic elements than in the quiet sun. One important consequence of neglecting NLTE is that the present models cannot reproduce the onset of the chromospheric temperature rise. Since the source functions in the cores of the strongest low excitation lines, which are formed highest in the atmosphere, begin to decouple from the Planck function, in LTE calculations the temperature will generally have to keep dropping with height to mimic the source function.

\section{Velocities: Flows, Oscillations and Waves}

Mass-motions in all solar photospheric features are studied with basically similar techniques. Some of the generally used diagnostics in the quiet sun and in sunspots are: the wavelength of the Stokes $I$ line core, the line width, the line asymmetry and the time dependence of these parameters. In complete analogy the diagnostics of mass-motions in and around magnetic elements are: The zero-crossing wavelength, line width and asymmetry of Stokes $V$ and the temporal variation of these quantities. ${ }^{*}$ The Stokes $V$ diagnostics can, naturally, also be used in sunspots. Conversely the Stokes $I$ velocity sensitive parameters can also provide

* The variation of the Stokes $V$ amplitude or wing area as a function of time has also been interpreted in terms of mass-motions which is analogous to looking at variations in the line depth of Stokes $I$. 
an idea of the combined velocity field of the magnetic elements and of their surroundings. The evolution of features on filtergrams or magnetograms may be used to trace horizontal motions, but these shall not be discussed here (cf. Martin, Title, these proceedings).

\subsection{Velocities in Sunspots}

The oldest known dynamic effect in magnetic features is the Evershed flow, an outflow of matter at photospheric heights in the sunspot penumbra which becomes an inflow in the penumbral chromosphere (inverse Evershed effect), while lines formed near the temperature minimum (e.g. $12 \mu$ emission lines, cf. Deming et al., 1988) do not show any Evershed effect.

Measurements in the wings of photospheric lines give sizeable velocities of 1-4 $\mathrm{km} \mathrm{s}^{-1}$ (Beckers, 1968; Abdussamatov and Krat, 1970; Ichimoto, 1987). However, line cores show shifts of typically only 0-1.5 $\mathrm{km} \mathrm{s}^{-1}$ depending on position in the penumbra and on the spectral line (e.g. Wiehr et al., 1986; Ichimoto, 1987). A combination of the two measurements implies an asymmetry in the Stokes $I$ line profiles which can only be explained by a vertical and/or horizontal velocity gradient. Evidence that the Evershed flow is not homogeneous but is concentrated in the darker filaments has been found by, e.g., Abdussamatov and Krat (1970) and Mamadazimov (1972). Since this represents a gradient across the field lines (in a horizontal direction) it is at least partly responsible for the asymmetry in Stokes $I$. However, it is probably not the only source of Stokes $I$ asymmetry, since the change in sign of the Evershed effect with height also suggests the presence of a vertical velocity gradient. Finally, the observations of broad-band circular polarization (see Sect. 5.3) imply that a gradient along the (inclined) field lines is also present. It is still not clear whether the Evershed flow disappears within $2-3^{\prime \prime}$ of the outer boundary of the penumbra (e.g., Wiehr et al., 1986) or whether it continues to be seen further out as well (e.g., Kuveler and Wiehr, 1985).

Umbral dots show sizeable blueshifts of 2-3 $\mathrm{km} \mathrm{s}^{-1}$ compared to the dark umbral core (Kneer, 1973; Pahlke, 1988). Umbral Stokes $V$ profiles are asymmetric which Pahlke (1988) interprets as being due to different flow velocities in umbral dots and the umbral core.

Both the umbra and the penumbra are rich in oscillations and waves. Their amplitudes, periods and, in some cases, propagation speeds can be directly determined. Umbral Oscillations at the photospheric level generally have amplitudes substantially smaller than $0.5 \mathrm{~km} \mathrm{~s}^{-1}$ and periods close to 5 minutes (e.g. Beckers and Schultz, 1972; Soltau et al., 1976; Kneer et al., 1981; Lites and Thomas, 1985; Abdelatif et al., 1986). Lines formed close to the temperature minimum still see power in the 5 minutes band, but also see oscillations with a period of 3 minutes, which is the main period observed in the chromosphere (Lites and Thomas, 1985). The oscillations can have slightly different periods in different parts of the umbra (Lites, 1986), suggesting the presence of more than one independently oscillating cell within an umbra. The observed oscillations are probably not the only non-stationary mass-motions in umbrae. Line broadenings give rms velocities of 1-2 $\mathrm{km} \mathrm{s}^{-1}$ (both vertical and horizontal, Beckers, 1976), suggesting that there may be considerable power at very small scales (perhaps in connection with umbral dots). Penumbral oscillations have also been observed (Beckers and Schultz, 1972; Musman et al., 1976; Balthasar et al., 1987; Lites, 1988) and Musman et al. (1976) have detected signs of outwards propagation (running penumbral waves). Balthasar et al. (1987) and Lites (1988) find evidence that the penumbral oscillations are aligned along the magnetic field. The power in both the umbral and the penumbral 5-minute oscillations is considerably lower than in the quiet photosphere.

\subsection{Velocities in Magnetic Elements}

Stationary flows within magnetic elements are diagnosed from time-averaged Stokes $V$ zero-crossing measurements. The watershed in such measurements came towards the middle of the present decade. Earlier measurements all showed downflows between 0.5 and $2 \mathrm{~km} \mathrm{~s}^{-1}$ (e.g. Giovanelli and Ramsay, 1971; Giovanelli and Brown, 1977; Harvey, 1977; Giovanelli and Slaughter, 1978; Wiehr, 1985; Scholier and Wiehr, 1985), while later observations do not show any significant downflows (Stenflo and Harvey, 1985; Solanki, 1986; Stenflo et al., 1987a, Wiehr, 1987; Solanki and Pahlke, 1988; Muglach, private communication). In particular Solanki (1986), from the analysis of the zero-crossings of a few hundred unblended lines, could set an upper limit of $\pm 250 \mathrm{~m} \mathrm{~s}^{-1}$ on the stationary flow velocity in magnetic elements. The most recent confirmation of an absence of downflows comes from measurements in the infrared. In a study currently underway 
Karin Muglach has looked at all unblended Fe I lines between 1.5 and $1.7 \mu$ and finds that the data are consistent, within the scatter, with an absence of a stationary flow. This removes the remaining uncertainty (due to the measurements of a single line, Fe I $15648.5 \AA$, by Harvey, 1977, and Stenflo et al., 1987b) surrounding the unshifted zero-crossing of spatially and temporally averaged Stokes $V$ profiles. Of course, local flows of short duration in either direction cannot be excluded, but their confirmation awaits good $\mathrm{S} / \mathrm{N}$ high spatial resolution observations.

The discrepancy between the older and the newer observations can be explained either by the improvement in instrumentation (see Harvey, private communication, with regard to Harvey, 1977), selection effects (Solanki and Pahlke, 1988, with regard to Scholier and Wiehr, 1985) and differences in spectral resolution (Solanki and Stenflo, 1986, with regard to the rest of the measurements showing downflows). The observations showing downflows in Stokes $V$ generally have a lower spectral resolution than those which do not. The presence of an asymmetry in Stokes $V$, with the blue wing of $V$ being stronger than its red wing for nearly all lines near disk centre (cf. Sect. 5.3) means that after the spectral smearing caused by the spectrograph such a profile is always redshifted. Since the Stokes $V$ asymmetry was unknown before the middle of the current decade, this instrumentally produced redshift was (auite naturally) falsely interpreted as due to a downflow within the magnetic elements.

The variation in the zero-crossing wavelength has been followed as a function of time by Giovanelli et al. (1978) and Wiehr (1985). They observed waves with periods close to $300 \mathrm{~s}$ and the former authors also found evidence for upward propagation. However, the amplitudes suggested by these studies are only $\approx 0.2-0.3 \mathrm{~km} \mathrm{~s}^{-1}$ in the upper photosphere, i.e. similar to those of photospheric umbral oscillations. If taken at face value, these numbers suggest that flux tube waves are insignificant for the energetics of the outer solar atmosphere. However, as we shall see below, this conclusion is probably false.

Another source of information on non-stationary mass motions within magnetic elements are the line widths of Stokes $V$ profiles. Solanki (1986) has studied line widths of Stokes $V$ profiles formed in magnetic elements at solar disk centre, while Pantellini et al. (1988) have analysed them at various positions on the disk. The results clearly show the presence of non-stationary motions in small magnetic elements with amplitudes larger than found in the quiet sun, both along the field lines and across them. Although the exact amplitudes may be somewhat affected by uncertainties in the detailed temperature structure, this main result appears well established (cf. Keller, these proceedings). The line broadenings cannot be caused by velocities outside the magnetic elements (Solanki, 1989).

The difference between the velocity amplitudes derived from the zero-crossing time series and from line broadening measurements is easily explained if the small size of magnetic elements is taken into account. If numerous magnetic elements are present in the resolution element and the waves or oscillations in them are not exactly in phase then the Stokes $V$ zero-crossing signal gets strongly smeared and becomes difficult to measure. Also, if the wavelength of the wave is less than the width of the contribution function of the Stokes $V$ profile (defined by Van Ballegooijen, 1985a, and Grossmann-Doerth et al., 1988a), then the wave amplitude seen in the Stokes $V$ zero-crossing is smaller than the true amplitude (Solanki and Roberts, 1989).

\subsection{Stokes V Asymmetry}

The final velocity diagnostic, the asymmetry of the Stokes $V$ profile, is discussed separately since its observation and interpretation in sunspots and in magnetic elements are closely related to each other and its causes in magnetic elements have only recently been clarified.

The first observational suggestion of an asymmetry in Stokes $V$ came from the discovery of broad band circular polarization in sunspots (Illing et al., 1974a), later confirmed by Illing et al. (1974b, 1975), Kemp and Henson (1983), Henson and Kemp (1984) and Makita (1986). These observations measure the net circular polarization averaged over many spectral lines. Recently broad band circular polarization has also been measured outside sunspots (Kemp et al., 1987). Spectrally resolved observations of the asymmetry of individual Stokes $V$ profiles formed in magnetic elements have been published by, e.g., Stenflo et al. (1984), Solanki and Stenflo $(1984,1985)$, Wiehr (1985) and Pantellini et al. (1988). Near solar disk centre the area and amplitude of the blue Stokes $V$ wing of most lines is larger than the area, respectively amplitude of the red wing. When moving towards the limb the sign of the area asymmetry changes, first for the stronger lines, later for the 
weaker ones. For magnetic elements (and probably also for sunspots) the source of the broad band circular polarisation is dominantly the area asymmetry of the Stokes $V$ profiles of individual spectral lines as shown by Müret et al. (1988).

Auer and Heasley (1978) demonstrated that the Stokes $V$ profiles of lines formed in LTE are antisymmetric in the absence of velocities. The mechanisms proposed to explain the area asymmetry of Stokes $V$ are based on the violation of one or the other of the above two assumptions. An approach based on departures from LTE has been taken by Kemp et al. (1984) and Landi Degl'Innocenti (1985). However, any quantitative application to a solar situation is formidably complicated and has so far not been attempted. Also, the rather high densities in the photosphere make it unlikely that the extremely strong domination by the radiation field (compared to collisions), required by this "atomic orientation" model, is present. The fact that the Stokes $V$ profiles of Fe II lines, formed very close to LTE, are just as asymmetric as Fe I lines also speaks strongly against this mechanism. The use of velocities appears more promising. Illing et al. (1975) first showed that a velocity gradient along the line of sight, combined with a gradient in the magnetic field strength, produces an asymmetry (cf. Grigorjev and Katz, 1975). Auer and Heasley (1978) later pointed out that the magnetic field gradient is not required if the magnetic vector is not parallel to the line of sight.

Further work on sunspots has concentrated mainly on approximately reproducing the spacial distribution, the absolute level and the CLV of the measured broad band circular polarization (e.g. Landmann and Finn, 1979; Makita, 1986; Skumanich and Lites, 1987). Qualitative agreement with the observations of the spatial dependence of the broad band circular polarization of Henson and Kemp (1984), which show a maximum of the asymmetry near the inner border of the penumbra, can be reached within the confines of a self-similar sunspot model (Schlüter-Temesvary theory) with a considerable Evershed flow. The Evershed flow is therefore currently expected to be the main contributer to the $V$ asymmetry. The spectral dependence of the broad band circular polarization is mainly a result of the spectral density of absorption by lines (Mürset et al., 1988). A more thorough analysis awaits better data.

In magnetic elements the interpretations and models have tried to reproduce the observed Stokes $V$ line profiles. Obtaining the asymmetry without producing a zero-crossing shift in Stokes $V$ has been one of the main problems. Sánchez Almeida et al. (1988a, 1989) have found that they can reproduce the Stokes $V$ asymmetry and zero-crossing of a number of lines with a flow within the magnetic elements if they assume a field whose strength increases with height. However, such a model contradicts directly measured field strengths (Sect. 3.2). On the other hand, Solanki and Pahlke (1988) have shown that an intemal stationary downflow in a physically reasonable flux tube model, which satisfies observations of the magnetic field structure, produces synthetic spectra that conflict with the observations in various ways.

Van Ballegooijen (1985c) suggested that downflows outside the magnetic elements should produce the proper sign of the asymmetry. Grossmann-Doerth et al. (1988b, 1989) proved that in this case, i.e. when velocity and $B$ do not overlap, the Stokes $V$ profile is asymmetric but its zero-crossing wavelength corresponds exactly to the rest wavelength (cf. Fig. 6). One implication of this result is that 2-D models incorporating the expansion of the magnetic elements with height are necessary to reproduce the Stokes $V$ area asymmetry. Another implication is that the Stokes $V$ area asymmetry provides an excellent diagnostic of the velocity in the immediate surroundings of magnetic elements. Solanki (1989) has studied these diagnostic capabilities at disk centre in greater detail. He finds that for a 2-D flux tube model fulfilling the observational constraints on field strength and temperature, the area asymmetry of four lines with widely different properties can be satisfied by an external downflow of approximately $1 \mathrm{~km} \mathrm{~s}^{-1}$, if the immediate surroundings of the flux tube are $250-350 \mathrm{~K}$ cooler than the quiet sun. This conforms very well to the picture, derived from white light images and magnetograms, of magnetic elements being concentrated in cool, downflowing intergranular lanes (e.g. Dunn and Zirker, 1974, Mehltretter, 1974; Muller, 1983; Title et al., 1987).

However, Solanki (1989) also found that a velocity outside the flux tube alone cannot reproduce the shape and width of Stokes $V$ completely. In order to reproduce both these quantities, without affecting the zero-crossing wavelength too strongly, longitudinal waves or oscillations inside the magnetic elements, with a slight difference between the upflowing and downflowing phase had to be introduced. The data require wave amplitudes between 1 and $3.0 \mathrm{~km} \mathrm{~s}^{-1}$. Further information on the nature of the wavelike motion or more precise values of the amplitudes cannot be derived from the very simple model used (two velocity components only). 
Fig. 6: Schematic illustration using a two layered atmosphere of how an asymmetric Stokes $V$ profile may be produced without any shift of its zero-crossing wavelength. Only the special case of a magnetic field aligned along the line of sight is considered here. Bottom frame: Profiles of $\eta_{ \pm}$(absorption coefficients for right and left circularly polarized light, respectively) in the lower atmospheric layer, where there is no field but a downflow (the two profiles are identical and redshifted). Second lowest frame: $\eta_{ \pm}$in the upper layer where there is a magnetic field but no velocity. Third lowest frame: $I_{ \pm}$, the emergent intensity profiles for the two polarizations. Due to saturation effects $I_{+}$ has a larger equivalent width ( $\eta_{-}$in both atmospheric layers lies at almost the same wavelength, while $\eta_{+}$ is at widely different wavelengths). Topmost frame: $V=I_{+}-I_{-}$, with a larger area of the blue wing than of the red wing. Note that since in both the upper and lower atmosperic layers $\eta_{+}=\eta_{-}$at $\lambda_{0}, V\left(\lambda_{0}\right)=0$, i.e. $V$ is asymmetric, but unshifted.
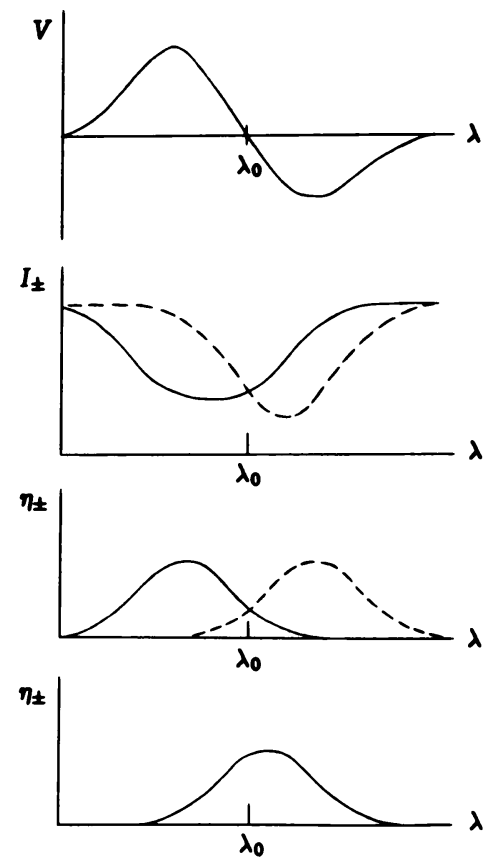

In summary, the Stokes $V$ profiles observed in magnetic elements at solar disk centre can be reproduced by a flux tube model with the field fanning out with height, having no stationary internal flow, but waves or oscillations with different up- and downflow phases. The flux tube is surrounded by a ring of cool downflowing gas. The change in sign of the asymmetry closer to the limb is probably due to the surrounding gas flowing horizontally towards the flux tubes (horizontal component of granular motion).

Acknowledgements: It is a pleasure to thank Dr. Gurtovenko and the other members of the Local Organising Committee for organising and financing my travel and stay in the USSR. Hania Allen kindly helped with the drawing of some of the figures, while David Evans and Alan Miles helped me to find the relevant literature on umbral and penumbral oscillations and waves.

\section{References}

Abdelatif, T.E., Lites, B.W., Thomas, J.H.: 1986, Astrophys. J. 311, 1015

Abdussamatov, H.I.: 1976, Solar Phys. 48, 117

Abdussamatov, H.I., Krat, V.A.: 1970, Solar Phys. 14, 132

Adjabshirzadeh, H., Koutchmy, S.: 1983, Astron. Astrophys. 122, 1

Albregtsen, F., Jorås, P.B., Maltby, P.: 1984, Solar Phys. 90, 17

Albregtsen, F., Maltby, P.: 1978, Nature 274, 41

Auer, L.H., Heasley, J.N.: 1978, Astron. Astrophys. 64, 67

Balthasar, H., Küveler, G., Wiehr, E.: 1987, Solar Phys. 112, 37

Beckers, J.M.: 1968, Solar Phys. 3, 258

Beckers, J.M.: 1976, Astrophys. J. 203, 739-752.

Beckers, J.M., Schröter, E.H.: 1968, Solar Phys. 4, 303

Beckers, J.M., Schröter, E.H.: 1969, Solar Phys. 10, 384 
Beckers, J.M., Schultz, R.B.: 1972, Solar Phys. 27, 61

Brandt, P.N., Solanki, S.K.: 1989, Astron. Astrophys. submitted

Brants, J.J., Zwaan, C.: 1982, Solar Phys. 80, 251

Brault, J.W., Noyes, R.W.: 1983, Astrophys. J. 269, L61

Buurman, J.: 1973, Astron. Astrophys. 29, 329

Chang, E.S., Noyes, R.W.: 1983, Astrophys. J. 275, L11

Chapman, G.A.: 1970, Solar Phys. 14, 315

Chapman, G.A.: 1974, Astrophys. J. 191, 255

Chapman, G.A.: 1977, Astrophys. J. Suppl. Ser. 33, 35

Chapman, G.A.: 1979, Astrophys. J. 232, 923

Del Toro Iniesta, J.C., Semel, M., Collados, M., Sánchez Almeida, J.: 1989, Astron. Astrophys. in press.

Deming, D., Boyle, R.J., Jennings, D.E., Wiedemann, G.: 1988, Astrophys. J. 333, 978

Deubner, F.L.: 1975, Osserv. Mem. Oss. Astrofis. Arcetri 105, 39

Deubner, F.L., Göhring, R.: 1970, Solar Phys. 13, 118

Dunn, R.B., Zirker, J.B.: 1973, Solar Phys. 33, 281

Foukal, P., Fowler, L.: 1984, Astrophys. J. 281, 442

Frazier, E.N., Stenflo, J.O.: 1978, Astron. Astrophys. 70, 789

Gingerich, O., Noyes, R.W., Kalkofen, W., Cuny, Y.: 1971, Solar Phys. 18, 347

Giovanelli, R.G., Brown, N.: 1977, Solar Phys. 52, 27

Giovanelli, R.G., Livingston, W.C., Harvey, J.W.: 1978, Solar Phys. 59, 49

Giovanelli, R.G., Ramsay, J.V.: 1971, in Solar Magnetic Fields, R. Howard (Ed.), IAU Symp. 43, 293

Giovanelli, R.G., Slaughter, C.: 1978, Solar Phys. 57, 255

Grigorjev, V.M., Katz, J.M.: 1975, Solar Phys. 42, 21

Grossmann-Doerth, U., Larsson, B., Solanki, S.K.: 1988a, Astron. Astrophys. 204, 266

Grossmann-Doerth, U., Schmidt, W.: 1981, Astron. Astrophys. 95, 366

Grossmann-Doerth, U., Schmidt, W., Schröter, E.H.: 1986, Astron. Astrophys. 156, 476

Grossmann-Doerth, U., Schüssler, M., Solanki, S.K.: 1988b, Astron. Astrophys. 206, L37

Grossmann-Doerth, U., Schüssler, M., Solanki, S.K.: 1989, Astron. Astrophys. in press

Gurman, J.B., House, L.L.: 1981, Solar Phys. 71, 5

Hagyard, M.J., Teuber, D., West, E.A., Tandberg-Hanssen, E., Henze, W., Beckers, J.M., Bruner, M., Hyder, C.L., Woodgate, B.E.: 1983, Solar Phys. 84, 13

Hartmann, L.: 1987, in Cool Stars, Stellar Systems, and the Sun, V., J.L. Linsky, R.E. Stencel (Eds.), Lecture Notes in Physics Vol. 291, Springer-Verlag, Berlin, p. 1

Harvey, J.W: 1977, in Highlights of Astronomy, E.A. Müller (Ed.), Vol. 4, Part II, p. 223

Harvey, J.W., Hall, D.: 1975, Bull. Amer. Astron. Soc. 7, 459.

Henson, G.D., Kemp, J.C.: 1984, Solar Phys. 93, 289

Hirayama, T.: 1978, Publ. Astron. Soc. Japan 30, 337

Hirayama, T., Hamana, S., Mizugaki, K.: 1985, Solar Phys. 99, 43

Ichimoto, K.: 1987, Publ. Astron. Soc. Japan 39, 329

Illing, R.M.E., Landman, D.A., Mickey, D.L.: 1974a, Astron. Astrophys. 35, 327

Illing, R.M.E., Landman, D.A., Mickey, D.L.: 1974b, Astron. Astrophys. 37, 97

Illing, R.M.E., Landman, D.A., Mickey, D.L.: 1975, Astron. Astrophys. 41, 183

Ioshpa, B.A., Obridko, V.N.: 1965, Soln. Dannye 3, 54

Kawakami, H.: 1983, , Publ. Astron. Soc. Japan 35, 459

Keller, C.U.: 1988, Diplomarbeit, E.T.H. Zürich.

Keller, C.U., Tarbell, T.D., Title, A.M., Solanki, S.K., Stenflo J.O.: 1989, Astron. Astrophys. submitted

Kemp, J.C., Henson, G.D.: 1983, Astrophys. J. 266, L69

Kemp, J.C., Henson, G.D., Steiner, C.T., Powell, E.R.: 1987a, Nature 326, 270

Kemp, J.C., Macek, J.H., Nehring, F.W.: 1984, Astrophys. J. 278, 863

Kjeldseth-Moe, O., Maltby, P.: 1974, Solar Phys. 35, 101

Kneer, F.: 1973, Solar Phys. 28, 361

Kneer, F., Mattig, W., Von Uexküll, M.: 1981, Astron. Astrophys. 102, 147 
Knölker, M., Schüssler, M.: 1988, Astron. Astrophys. 202, 275

Kollatschny, W., Stellmacher, G., Wiehr, E., Falipou, M.A.: 1980, Astron. Astrophys. 86, 245

Koutchmy, S.: 1977, Astron. Astrophys. 61, 397

Koutchmy, S., Adjabshirzadeh, A.: 1981, Astron. Astrophys. 99, 111

Koutchmy, S., Stellmacher, G.: 1978, Astron. Astrophys. 67, 93

Krat, V.A., Karpinsky, V.N., Pravidjuk, L.M.: 1972, Solar Phys. 26, 305

Küveler, G., Wiehr, E.: 1985, Astron. Astrophys. 142, 205

Landi Degl'Innocenti E.: 1985, in Theoretical Problems in High Resolution Solar Physics, H.U. Schmidt (Ed.), Max Planck Inst. f. Astrophys., Munich, p. 162

Landman, D.A., Finn, G.D.: 1979, Solar Phys. 63, 221

Lemke, M. Holweger, H.: 1987, Astron. Astrophys. 173, 375

Lites, B.W.: 1986, Astrophys. J. 301, 992

Lites, B.W.: 1988, Astrophys. J. 334, 1054

Lites, B.W., Scharmer, G.: 1989, in High Spatial Resolution Solar Observations, O. Von der Lühe (Ed.), Sacramento Peak, NM, in press.

Lites, B.W., Skumanich, A.: 1989, Astrophys. J. in press

Lites, B.W., Thomas, J.H.: 1985, Astrophys. J. 294, 682

Makita, M.: 1986, Solar Phys. 106, 269

Maltby, P.: 1972, Solar Phys. 26, 76

Maltby, P., Avrett, E.H., Carlsson, M., Kjeldseth-Moe, O., Kurucz, R.L., Loeser, R.: 1986, Astrophys. J. 306, 284

Mamadazimov, M.: 1972, Solar Phys. 22, 129

Martin, S.F.: 1988, Solar Phys. 117, 243

Mattig, W.: 1961, Mitt. Astron. Gesell. Hamburg 14, 47

Mehltretter, J.P.: 1974, Solar Phys. 38, 43

Muller, R.: 1973, Solar Phys. 32, 409

Muller, R.: 1983, Solar Phys. 85, 113

Muller, R., Keil, S.L.: 1983, Solar Phys. 87, 243

Mürset, U., Solanki, S.K., Stenflo, J.O.: 1988, Astron. Astrophys. 204, 279

Musman, S., Nye, A.H., Thomas, J.H.: 1976, Astrophys. J. 206, L175

Obridko, V.N.: 1968, Bull. Astron. Inst. Chechoslovakia 19, 183

Obridko V.N., Staude, J.: 1988, Astron. Astrophys. 189, 232

Pahlke, K.-D.: 1988, Ph.D. Thesis, University of Göttingen

Pantellini, F.G.E., Solanki, S.K., Stenflo, J.O.: 1988, Astron. Astrophys. 189, 263

Rachkovsky, D.N., Tsap, T.T.: 1985, Izv. Krymskoj Astrofiz. Obs. 71, 79

Rees, D.E., Semel, M.D.: 1979, Astron. Astrophys. 74, 1

Robinson, R.D.: 1980, Astrophys. J. 239, 961

Robinson, R.D., Worden, S.P., Harvey, J.W.: 1980, Astrophys. J. 236, L155

Saar, S.H.: 1988, Astrophys. J. 324, 441

Sánchez Almeida, J., Collados, M., Del Toro Iniesta, J.C.: 1988a, Astron. Astrophys. 201, L37

Sánchez Almeida, J., Collados, M., Del Toro Iniesta, J.C.: 1989, Astron. Astrophys. in press

Sánchez Almeida, J., Solanki, S.K., Collados, M., del Toro Iniesta, J.C.: 1988b, Astron. Astrophys. 196, 266

Scholiers, W., Wiehr, E.: 1985, Solar Phys. 99, 349

Schüssler, M.: 1986, in Small Scale Magnetic Flux Concentrations in the Solar Photosphere, W. Deinzer, M. Knölker, H.H. Voigt (Eds.), Vandenhoeck \& Ruprecht, Göttingen, p. 103

Schüssler, M., Solanki, S.K.: 1988, Astron. Astrophys. 192, 338

Shine, R.A., Linsky, J.L.: 1974, Solar Phys. 37, 145

Schmahl, G.: 1967, Z. Astrophys. 66, 81

Skumanich, A., Lites, B.W.: 1987, Astrophys. J. 322, 483

Sobotka, M.: 1988, Bull. Astron. Inst. Czechoslovakia 39, 236

Sobotka, M.: 1989, Solar Phys. in press

Solanki, S.K.: 1986, Astron. Astrophys. 168, 311 
Solanki, S.K.: 1989, Astron. Astrophys. in press

Solanki, S.K., Keller, C., Stenflo, J.O.: 1987, Astron. Astrophys. 188, 183

Solanki, S.K., Pahlke, K.D.: 1988, Astron. Astrophys. 201, 143

Solanki, S.K., Roberts, B.: 1989, in Proc. Chapman Conference on Magnetic Flux Ropes, C.T. Russell (Ed.), in press

Solanki, S.K., Steenbock, W.: 1988, Astron. Astrophys. 189, 243

Solanki, S.K., Stenflo, J.O.: 1984, Astron. Astrophys. 140, 185

Solanki, S.K., Stenflo, J.O.: 1985, Astron. Astrophys. 148, 123

Solanki, S.K., Stenflo, J.O.: 1986, Astron. Astrophys. 170, 120

Solanki, S.K., Zayer, I., Stenflo, J.O.: 1989, in High Spatial Resolution Solar Observations, O. Von der Lühe (Ed.), Sacramento Peak, NM, in press.

Soltau, D., Schröter, E.H., Wöhl, H.: 1976, Astron. Astrophys. 50, 367

Stellmacher, G., Wiehr, E.: 1973, Astron. Astrophys. 29, 13

Stellmacher, G., Wiehr, E.: 1979, Astron. Astrophys. 75, 263

Stenflo, J.O.: 1973, Solar Phys. 32, 41

Stenflo, J.O.: 1975, Solar Phys. 42,79

Stenflo, J.O.: 1988, Solar Phys. 114, 1

Stenflo, J.O.: 1989, Astron. Astrophys. Rev. 1, 3.

Stenflo, J.O., Harvey, J.W.: 1985, Solar Phys. 95, 99

Stenflo, J.O., Harvey, J.W., Brault, J.W., Solanki, S.K.: 1984, Astron. Astrophys. 131, 333

Stenflo, J.O., Solanki, S.K., Harvey, J.W.: 1987a, Astron. Astrophys. 171, 305

Stenflo, J.O., Solanki, S.K., Harvey, J.W.: 1987b, Astron. Astrophys. 173, 167

Stenholm, L.G., Stenflo, J.O.: 1978, Astron. Astrophys. 67, 33

Stepanov V.E.: 1965, IAU Symp. 22, 267

Sun, W.-H., Giampapa, M.S., Worden, S.P.: 1987, Astrophys. J. 312, 930

Tarbell, T.D., Title, A.M.: 1977, Solar Phys. 52, 13

Title, A.M., Tarbell, T.D., Topka, K.P.: 1987, Astrophys. J. 317, 892

Van Ballegooijen, A.A.: 1984, Solar Phys. 91, 195

Van Ballegooijen, A.A.: 1985a, in Measurements of Solar Vector Magnetic Fields, M.J. Hagyard (Ed.), NASA Conf. Publ. 2374, p. 322

Van Ballegooijen, A.A.: 1985b, in Theoretical Problems in High Resolution Solar Physics, H.U. Schmidt (Ed.), Max Planck Inst. f. Astrophys., Munich, p. 167

Van Ballegooijen, A.A.: 1985c, in Theoretical Problems in High Resolution Solar Physics, H.U. Schmidt (Ed.), Max Planck Inst. f. Astrophys., Munich, p. 177.

Vernazza, J.E., Avrett, E.H., Loeser, R.: 1981, Astrophys. J. Suppl. Ser. 45, 635

Von der Lühe, O.: 1989, in High Spatial Resolution Solar Observations, O. Von der Lühe (Ed.), Sacramento Peak, NM, in press.

Walton, S.R.: 1987, Astrophys. J. 312, 909

Wiehr, E.: 1978, Astron. Astrophys. 69, 279

Wiehr, E.: 1985, Astron. Astrophys. 149, 217

Wiehr, E.: 1987, in Cool Stars, Stellar Systems, and the Sun, V., J.L. Linsky, R.E. Stencel (Eds.), Lecture Notes in Physics Vol. 291, Springer-Verlag, Berlin, p. 54

Wiehr, E., Stellmacher, G.: 1985, in High Resolution in Solar Physics, Lect. Notes in Phys. 233, 254

Wiehr, E., Stellmacher, G., Knölker, M., Grosser, H.: 1986, Astron. Astrophys. 155, 402

Wittmann, A.D.: 1971, Solar Phys. 20, 365

Wittmann, A.D.: 1974, Solar Phys. 36, 29

Zayer, I., Solanki, S.K., Stenflo, J.O.: 1988, Astron. Astrophys. in press

Zirin, H., Popp, B.: 1989, Astrophys. J. in press

Zwaan, C., Brants, J.J., Cram, L.E.: 1985, Solar Phys. 95, 3 